\title{
14 Controller Switching Strategy for Constrained Systems and Its Application to Hard Disk Drives* $^{*}$
}

\author{
Atsushi OKUYAMA** and Takashi YAMAGUCHI ${ }^{* * *}$ \\ ** Department of Precision Engineering, Tokai University \\ 1117 Kitakaname, Hiratsuka, Kanagawa 259-1292, Japan \\ *** Hitachi Global Storage Technologies, Inc. \\ 1 Kirihara, Fujisawa, Kanagawa 252-8588, Japan
}

\begin{abstract}
We propose a switching control strategy for systems with state and control constraints. Prior studies have proven that switching control strategies have the ability to meet performance objectives, such as fast response and good disturbance rejections, while avoiding constraint violations. The controller is selected on-line from a given set of controllers by supervisory rules based on the concept of a maximal output admissible set. The selected controller needs to be appropriately initialized during switching, but how to decide the controller's initial state is still a problem. This paper proposes a method that utilizes an initial value compensation (IVC) technique for determining the initial state of the controller. The IVC technique has the following features. First, the controller's initial state is chosen according to the plant's initial state. Therefore, the number of variables that the supervisory rules need to consider is reduced. Second, smooth and fast transient responses can be obtained after switching, therefore the region of the closed-loop initial state variables that satisfy the desired response specifications under the given constraints can be expanded. Experimental evaluations of the proposed switching control strategy were performed with a 2.5 -inch form-factor hard disk drive.
\end{abstract}

Key words : Hard Disk Drive, Constrained System, Switching Control

\section{Introduction}

Constraints on the control inputs and the state variables are common in practical control systems. Violations of such constraints drastically degrade system performances and lead to instability in worst-case scenarios. To avoid these reductions in performance, switching control strategy techniques have been proposed ${ }^{(3)-(6)}$. The purpose of the switching control strategy is to meet the performance objectives, such as fast response and good disturbance rejections, which satisfy the specified states and control constraints.

In synthesizing the switching control strategies, utilizing a maximal output admissible set $^{(1)-(6)}$ is important for ensuring the safe operation of the system for the given state and control constraints. The maximal output admissible set is a set of all closed-loop initial states, which guarantees to satisfy the given constraints. For full state feedback, supervisory rules for safe operations can be determined by using the maximal output admissible set. For the dynamic controller, however, the question of how to decide the controller's initial state remains a problem that needs to be solved.

In this paper, we consider a dynamic controller switching strategy based on the maximal output admissible set. The main idea is to utilize an initial value compensation (IVC) technique $^{(7)-(16)}$ for determining the controller's initial state. The initial value compensation technique has the following features. First, the controller's initial state is chosen by the plant's 


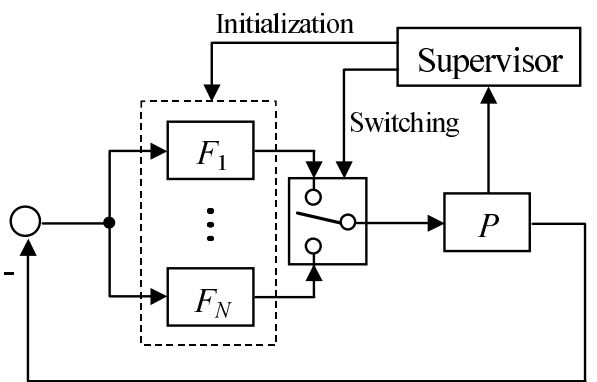

Fig. 1 Switching Control System

initial state, i.e., the plant initial state variables that represent the whole closed-loop initial state variables. Therefore, the number of variables that the supervisory rules need to consider is reduced. Second, smooth and fast transient responses can be obtained after switching, thus the region of the closed-loop initial state variables that satisfy the desired response specifications under given constraints can be expanded. Experimental evaluations were performed for the proposed switching control strategy with a 2.5 -inch form-factor hard disk drive (HDD).

\section{Problem Formulation}

\subsection{Switching Control System}

Fig. 1 is a block diagram of a switching control system. The plant dynamics under consideration are

$$
P:\left\{\begin{array}{l}
x_{p}[k+1]=A_{p} x_{p}[k]+B_{p} u[k] \\
y[k]=C_{p} x_{p}[k]
\end{array},\right.
$$

where $x_{p} \in R^{n}$ is the state vector of the plant, $u \in R^{m}$ is the control input, $y \in R^{p}$ is the control output. Here, $k$ is the discrete-time index. $A_{p}, B_{p}$, and $C_{p}$ are the real matrices with appropriate dimensions. The state and control input constraints are described as

$$
z[k]=C_{x} x_{p}[k]+C_{u} u[k] \in Z, k \geq 0,
$$

where $z \in R^{q}$ is the vector signal to be constrained, $C_{x}$ and $C_{u}$ are the real matrices with appropriate dimensions. $Z$ is the following convex polyhedral set,

$$
Z=\left\{z \mid M_{z} z \leq m_{z}\right\}, M_{z} \in R^{r \times q}, m_{z} \in R^{r} .
$$

The family of switched controllers under consideration is also a set of linear discrete-time systems

$$
F_{i}:\left\{\begin{array}{l}
x_{c i}[k+1]=A_{c i} x_{c i}[k]+B_{c i}(-y[k]) \\
u[k]=C_{c i} x_{c i}[k]+D_{c i}(-y[k])
\end{array},\right.
$$

where $x_{c i} \in R^{n_{c i}}$ is the state vector of each controller, subscription $i \in\{1,2, \ldots, N\}$ denotes the index of the $i$ th controller, and $A_{c i}, B_{c i}, C_{c i}$, and $D_{c i}$ are the real matrices with appropriate dimensions. The closed-loop system, which consists of the plant and the $i$ th controller, is

$$
\left\{\begin{array}{l}
x_{i}[k+1]=A_{i} x_{i}[k] \\
y[k]=C_{y i} x_{i}[k] \\
z[k]=C_{z i} x_{i}[k]
\end{array},\right.
$$

where $x_{i}=\left[\begin{array}{ll}x_{p}^{T} & x_{c i}^{T}\end{array}\right]^{T} \in R^{n_{i}}$ is the state vector of each closed-loop system and $n_{i}=n+n_{c i}$. The matrices in Eq. 5 are given as

$$
\begin{aligned}
A_{i} & =\left[\begin{array}{cc}
A_{p}-B_{p} D_{c i} C_{p} & B_{p} C_{c i} \\
-B_{c i} C_{p} & A_{c i}
\end{array}\right], \\
C_{y i} & =\left[\begin{array}{ll}
C_{p} & 0_{p \times n_{c i}}
\end{array}\right], \text { and } \\
C_{z i} & =\left[\begin{array}{ll}
C_{x}-C_{u} D_{c i} C_{p} & C_{u} C_{c i}
\end{array}\right] .
\end{aligned}
$$




\subsection{Maximal Output Admissible Set}

The vector signal to be constrained at time $k$ can be expressed as

$$
z[k]=C_{z i} A_{i}^{k} x_{i}[0] .
$$

Thus, the maximal output admissible set is defined as

$$
O_{\infty}^{i}=\left\{x_{i}[0] \mid z[k] \in Z, k \geq 0\right\} .
$$

The maximal output admissible set is a set of all closed-loop initial states, which guarantees to satisfy the constraints defined in Eq. 2. From the definition of the maximal output admissible set in Eq. 7, the $i$ th closed-loop system will operate by avoiding violations of the constraints if and only if $x_{i}[0] \in O_{\infty}^{i}$. The maximal output admissible set can be iteratively found by solving a finite number of linear programs ${ }^{(1),(2)}$.

\subsection{Controller Switching Strategy}

This is a brief review of the controller switching strategy under full state feedback ${ }^{(3),(4)}$. We assumed that a family of switched controllers had already been designed, whose closedloop systems are asymptotically stable, and the controllers were arranged in a given sequence to achieve good performance. The maximal output admissible sets of each closed-loop system are given. A supervisory rule selects the controller with the highest performance according to the following rule at time $k$

$$
i[k]=\text { maximize } i \text { subject to } x_{i}[k] \in O_{\infty}^{i} \text {. }
$$

To determine the controller, the supervisor establishes whether the current closed-loop state is contained within the maximal output admissible set of Eq. 7. For full state feedback, the state of each closed-loop system only becomes the state of the plant, i.e., $x_{i}[k]=x_{p}[k]$. Thus, the switching condition of the supervisory rule is simple. However, for the dynamic controller that we need to consider here, the closed-loop state contains the state of the dynamic controller. Thus, the switching condition for the supervisory rule becomes complicated. Furthermore, as the selected controller needs to be appropriately initialized during switching, how to determine the initial state of the dynamic controller remains a problem that needs to be solved. As previously mentioned, we propose a method that utilizes IVC to determine the initial state of the dynamic controller.

\section{Initial Value Compensation}

We assumed that the plant state could be measured. IVC produces the initial state of the dynamic controller according to the initial state of the plant as

$$
x_{c i}[0]=K_{i} x_{p}[0],
$$

where $K_{i}$ is the linear mapping matrix to be designed. Let us briefly review the design method based on the following linear-quadratic optimization ${ }^{(7),(8)}$ :

$$
\min _{x_{c i}[0]} J_{i}=\sum_{k=0}^{\infty} x_{i}^{T}[k] Q_{i} x_{i}[k], Q_{i}>0,
$$

where $k=0$ indicates the instant of switching and $Q_{i}$ is the weighting matrix.

By using the following discrete-time Lyapunov equation,

$A_{i}^{T} P_{i} A_{i}-P_{i}=-Q_{i}$,

Eq. 10 can be transformed into

$$
J_{i}=x_{i}^{T}[0] P_{i} x_{i}[0]
$$

and

$$
P_{i}=\left[\begin{array}{ll}
p_{11 i} & p_{12 i} \\
p_{21 i}^{T} & p_{22 i}
\end{array}\right],
$$




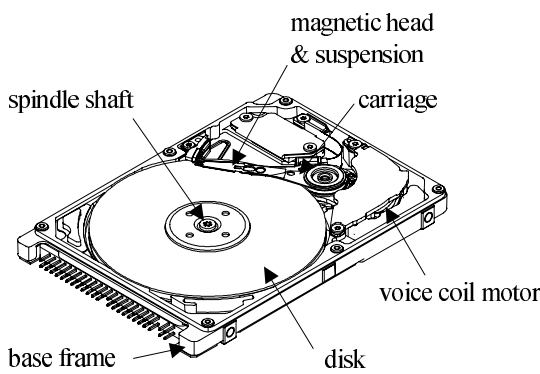

Fig. 2 Schematic diagram of a 2.5-inch HDD

where $P_{i}$ is the positive definite matrix to satisfy Eq. $11 . J_{i}$ is differentiated by $x_{c i}[0]$ to obtain a minimum value for it.

$$
\frac{\partial J_{i}}{\partial x_{c i}[0]}=2 p_{22 i} x_{c i}[0]+2 p_{12 i} x_{p}[0] .
$$

Therefore,

$$
x_{c i}[0]=K_{i} x_{p}[0], K_{i}=-p_{22 i}^{-1} p_{12 i} .
$$

The transient response of the closed-loop system after switching can be minimized using IVC in the sense that $J_{i}$ can be minimized. Furthermore, the initial state of the controller is produced by the initial state of the plant. Thus, the maximal output admissible set in Eq. 7 can be expressed as

$$
O_{\infty}^{i}=\left\{x_{p}[0] \mid z[k] \in Z, k \geq 0\right\}
$$

and

$$
z[k]=C_{z i} A_{i}^{k}\left[\begin{array}{c}
I \\
K_{i}
\end{array}\right] x_{p}[0] .
$$

The number of variables that the supervisory rules need to consider can be reduced as well as for full state feedback.

\section{Application to Hard Disk Drives}

\subsection{Servo System in Hard Disk Drives}

A schematic diagram of a 2.5-inch form factor HDD is shown in Fig. 2. Two disks are stacked on the spindle motor shaft and rotate at 4,200 revolutions per minute. On the surface of the disk, more than 10,000 data tracks are magnetically recorded. A suspension and a carriage support the head. An actuator, called the voice coil motor (VCM), actuates the carriage and moves the head to the desired track. The mechanical parts are the head, the disk, the spindle motor, the VCM, the suspension, and the carriage. The spindle motor and the VCM are mounted on a base frame. On the back of the head-disk assembly mechanism (HDA) is a circuit board on which a microprocessor or a digital signal processor (DSP) is mounted. The top of the HDA is covered by a top cover. The plant is modeled as a rigid-body model with a delay element, which is modeled as a first-order padé approximation. That is, the plant is a third order system as

$$
P(s)=\left(\frac{K}{s^{2}}\right)\left(\frac{-0.5 L s+1}{0.5 L s+1}\right),
$$

where $s$ is the Laplace operator, $L$ is the total time delay, and $K$ is the gain that includes the VCM torque constant, power amplifier gain, and other constants.

In an HDD servo system, mode-switching control (MSC) has commonly been used. There is a block diagram of an MSC system in Fig. 3(a). There are two modes: seeking and following. The seeking mode rapidly moves the head to the target track, and the following mode accurately maintains the head along the center of the track. Occasionally, a settling 


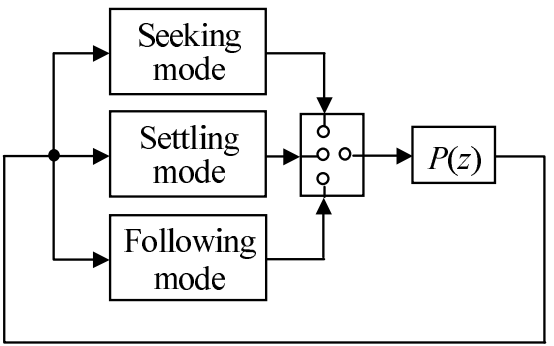

(a) Block diagram of mode switching control system

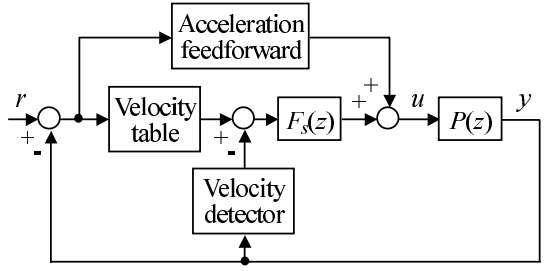

(b) Block diagram of seeking mode

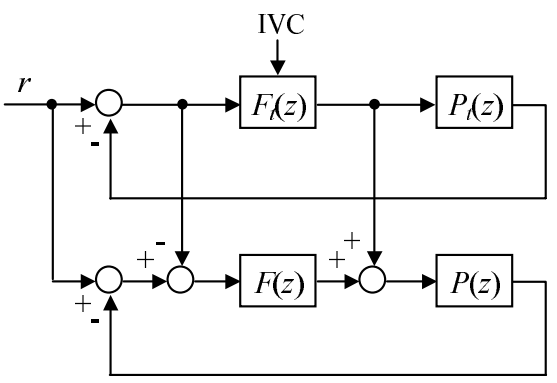

(c) Block diagram of model-following control with IVC

Fig. 3 Basic schematic diagrams of mode switching control system

mode to enable smooth accommodation onto the target track is added. Each servo mode is designed to independently satisfy a certain cost function. MSC is a kind of switching-control system. However, the order for the controller's switching is determined beforehand. That is, the controller is switched in order of seeking, settling, and following. Therefore, selection is not needed as the supervisory in MSC. Decisions on the controller's switching conditions and its initialization are needed as the supervisory rule does not select the controller in MSC.

The plant is controlled by velocity-based two-degree-of-freedom control in the seeking mode shown in Fig. 3(b). During acceleration, the power amplifier is saturated and the maximum current drives a voice coil motor. During deceleration, the reference velocity trajectory and the feedforward acceleration trajectory are generated as a function of position error, and the velocity servo is controlled within the linear part of the power amplifier.

The plant is controlled by model-following control with IVC in the settling and following modes shown in Fig. 3(c), where $F(z)$ is a following controller consisting of a phase-lead filter and a pseudo-integral element to reduce the steady-state error. Here, $F_{t}(z)$ is a settling controller designed with the pole placement method and $P_{t}(z)$ is a plant model (usually, a rigid-body one with a delay element). When mode-switching from the seeking to the settling mode, model-following control with IVC operates as follows: (1) IVC is applied to the settling controller; (2) 0 is set as the initial state of the following controller, and (3) the initial state of the actual plant is set as the initial state of the plant model. After mode-switching from the seeking to the settling mode, the plant is controlled by the settling controller with IVC. Then, in the upper settling control system shown in Fig. 3(c), positioning error and control input become 0 in the steady state. Consequently, when the mode switches to the following mode from the settling mode, the controller is continuously switched from the settling controller to the following controller. Therefore, mode switching can only be done once from the seeking 


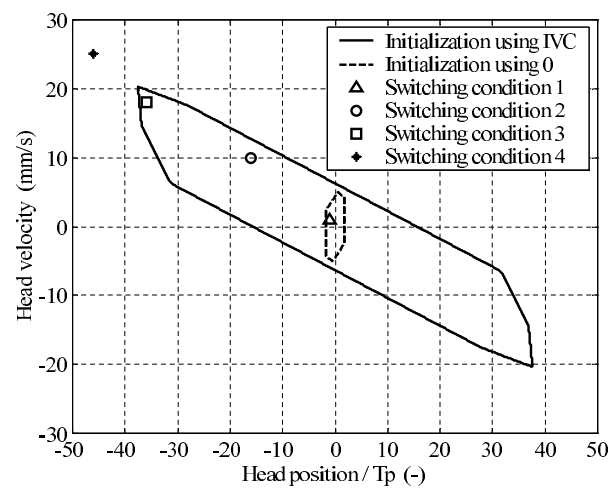

Fig. 4 Maximal output admissible sets

to the settling mode.

Consumer products containing HDDs, such as video recorders and audio jukeboxes, have also appeared on the market in recent years. The need to reduce acoustic noise has been increasing in HDDs with such diversified uses. One kind of acoustic noise in HDDs is seeking noise generated by seeking operation. Seeking noise has been assumed to originate from transient vibrations, not steady vibrations, in the mechanical parts ${ }^{(13)}$. Therefore, bumpless switching is needed to reduce this noise.

\subsection{Design Results}

Switching conditions from the seeking to the settling mode are designed as follows: (1) the settling controller is designed, (2) the linear mapping matrix for IVC is designed, (3) the maximal output admissible set is predicted so that the required response specifications are satisfied under the given constraints, and (4) the switching conditions, which mean the final state of the plant in the seeking mode, are determined by the predicted maximal output admissible set. In addition, since the purpose of the seeking mode is to move the plant state to the specified final state as quickly as possible, the seeking profile also needs to be redesigned according to the switching conditions. The settling controller was designed by pole placement. The poles of the closed-loop transfer function are placed at $0.24,0.25,0.26,0.50$, and 0.60 on the real axis of the real/imaginary plane. The sampling time is about $0.1 \mathrm{~ms}$.

We imposed the following constraints on the control input, $u=z \in R$, after switching, so that bumpy switching would not occur:

$$
-0.02 \leq u[k] \leq 0.02 \quad k \geq 0
$$

where control input $u$ is current to the VCM. The cost function of IVC was defined as follows to prevent bumpy switching:

$$
\min _{x_{c i}[0]} J_{i}=\sum_{k=0}^{\infty} u[k]^{2}=\sum_{k=0}^{\infty} x_{i}^{T}[k] Q_{i} x_{i}[k],
$$

and

$$
Q_{i}=\left[\begin{array}{c}
-\left(D_{c i} C_{p}\right)^{T} \\
C_{c i}^{T}
\end{array}\right]\left[\begin{array}{ll}
-D_{c i} C_{p} & C_{c i}
\end{array}\right]
$$

Figure 4 is the maximal output admissible set we predicted. Here, the state variable of the delay element in Eq. 16 is set to 0. The solid line is where the controller initial state is initialized by the IVC technique, and the dashed line is where the controller initial state is set to 0 . The horizontal axis is the head position normalized by the track-pitch Tp, and the vertical axis is the head velocity. The origin of the horizontal axis shows the target track position. For instance, -10 of the horizontal axis shows the position of 10 tracks forward from the target 


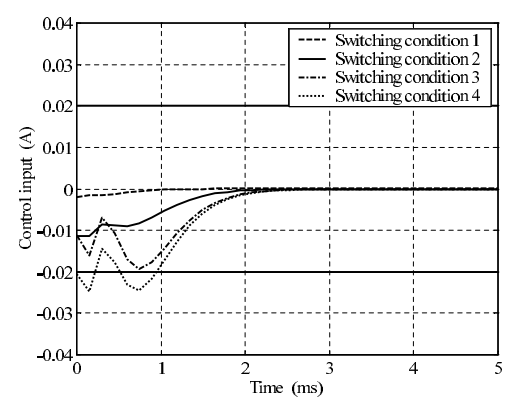

(a) Control input

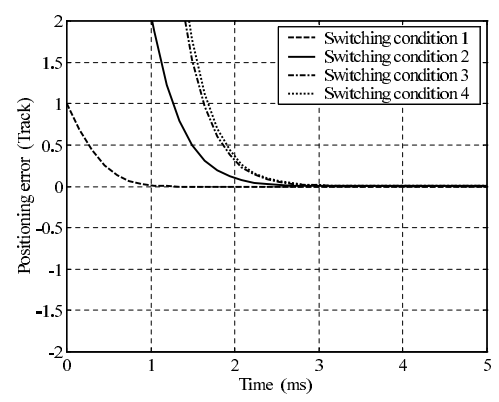

(b) Positioning error

Fig. 5 Initialization using IVC

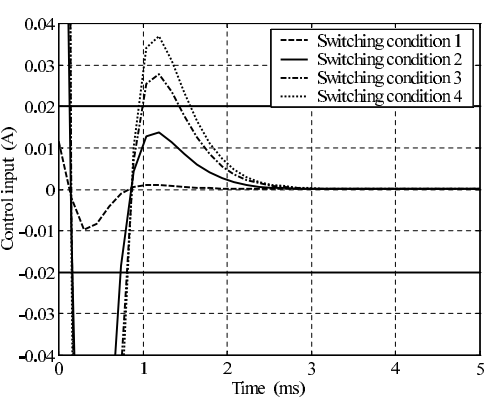

(a) Control input

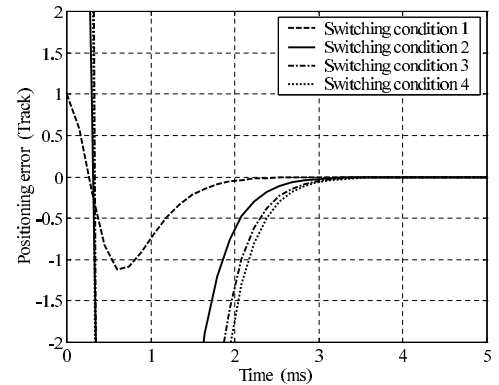

(b) Positioning error

Fig. 6 Initialization using 0

Table 1 Switching conditions

\begin{tabular}{c|c|c}
\hline Switching conditions & Head position (Track) & Head velocity $(\mathrm{mm} / \mathrm{s})$ \\
\hline 1 & -1 & 1 \\
2 & -16 & 10 \\
3 & -36 & 18 \\
4 & -46 & 25 \\
\hline
\end{tabular}

track. The safe region where the constraint is fulfilled is expanded using the IVC technique, as can be seen in Fig. 4.

Figures 5 and 6 are the simulation results. Initializations using IVC and 0 are shown in Figs. 5 and 6, respectively. The four switching conditions listed in Table 1 were evaluated for the simulation. The relation between the maximal output admissible set and the four switching conditions is shown in Fig. 4. For the initialization using IVC shown in Fig. 5, when the switching conditions are contained within the maximal output admissible set, the constraint on the control input is fulfilled. The transient response of the positioning error is good for all switching conditions. On the other hand, only switching condition 1 is contained within the maximal output admissible set for initialization using 0 in Fig. 4. The constraint on the control input is fulfilled only for switching condition 1 . However, even when the switching condition is contained within the maximal output admissible set, the transient response of the positioning error is not good. That is, an overshoot occurs.

\subsection{Experimental Results}

The experimental results for the initialization using IVC are shown in Fig. 7. Switching condition 2 listed in Table 1 is used. (a) is a plot of the whole control input waveform, (b) is a close-up of (a), and (c) is a plot of the positioning error waveform after switching to the settling mode from the seeking mode. The average, maximum, and minimum positioning error waveforms in a 200-times experiment are plotted. The constraint on the control input is fulfilled and the transient response of the positioning error is good. In addition, when the 


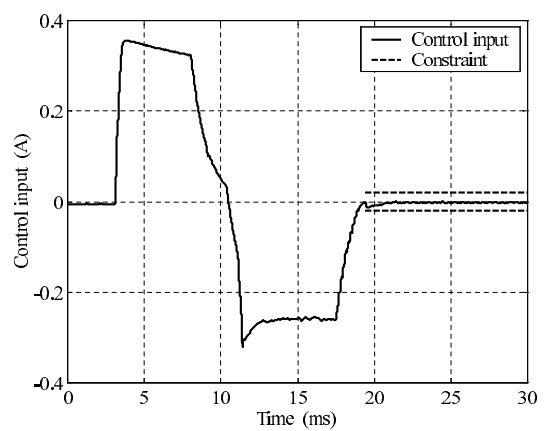

(a) Control input

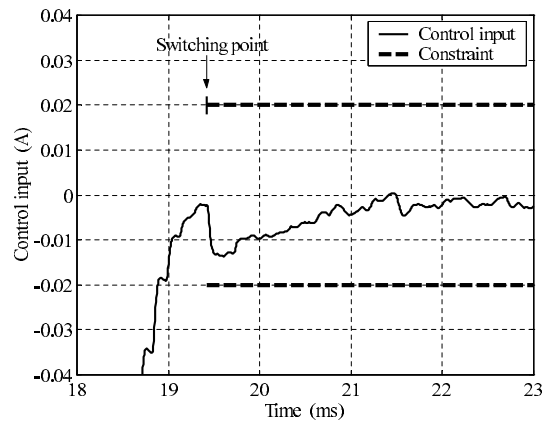

(b) Close-up of (a)

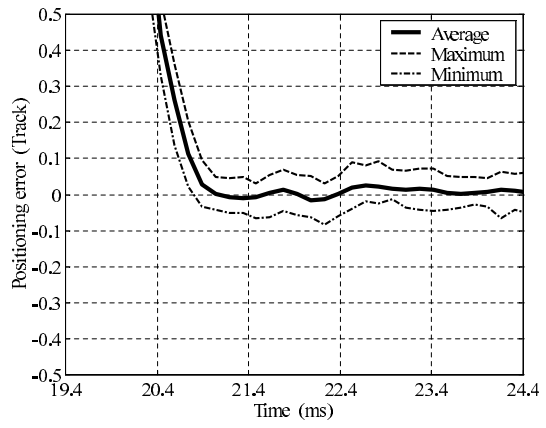

(c) Positioning error after switching

Fig. 7 Experimental results for initialization using IVC

controller initial state had been set to 0 and the switching condition 1 had been set to within the maximal output admissible set for initialization using 0 in Fig. 4, we experimentally confirmed that the constraints on control input were satisfied but large overshoot occurred in positioning error.

\section{Conclusion}

We propose a dynamic controller switching strategy for systems that have state and control constraints. The maximal output admissible set predicts the safe switching condition so that the given constraints are fulfilled. The plant state determines the initial state of the dynamic controller at the outset of switching by using the initial value compensation (IVC) technique. The transient response of the closed-loop system after the switching can be improved by using the IVC technique. Therefore, the region of the closed-loop initial state variables that satisfy the desired response specifications under the given constraints can be expanded. Tests with a 2.5-inch form-factor hard disk drive confirmed that the proposed controller switching strategy satisfies the given constraint on the control input and significantly improves the transient response after switching.

\section{References}

( 1 ) E. G. Gilbert and K. T. Tan: Linear systems with state and control constraints: The theory and application of maximal output admissible sets, IEEE Trans. on Automatic Control, Vol. 36, No. 9, pp. 1008-1020, 1991

( 2 ) K. Hirata and M. Fujita: Set of admissible reference signals and control of systems with state and control constraints, In Proceedings of the 38th IEEE Conference on Decision and Control, pp. 1427-1432, 1999.

( 3 ) K. Hirata and M. Fujita: Measurement feedback switching control for systems with state and control constraints, In Proceedings of the 2000 American Control Conference, pp. 3321-3325, 2000.

( 4 ) K. Hirata and M. Fujita: Observer based switching control for systems with state and 
control constraints, In Proceedings of the 2001 American Control Conference, pp. 18921897, 2001.

( 5 ) K. Kogiso and K. Hirata: Controller switching strategies for constrained mechanical systems with applications to the remote control over networks, In Proceedings of the 2004 CCA/ISIC/CACSD, pp. 480-484, 2004.

( 6 ) K. Kogiso, T. Matsumoto and K. Hirata: Experimental validation of controller switching strategies for constrained systems, In Proceedings of the SICE Annual Conference 2005, 2005.

( 7 ) T. Yamaguchi, K. Shishida, H. Hirai, K. Tsuneta and M. Sato: Improvement of servo robustness for digital sector servo system, IEEE Trans. on Magnetics, Vol. 28, No. 5, pp. 2910-2912, 1992.

( 8 ) T.Yamaguchi, K.Shishida, S. Tohyama, and H. Hirai: Mode Switching Control Design with Initial Value Compensation and Its Application to Head Positioning Control on Magnetic Disk Drives, IEEE Trans. on Industrial Electronics, Vol. 43, No. 1, pp. 65-73, 1996.

( 9 ) T.Yamaguchi, Y.Soyama, H.Hosokawa, K.Tsuneta, and H. Hirai: Improvement of Settling Response of Disk Drive Head Positioning Servo using Mode Switching Control with Initial Value Compensation, IEEE Trans. on Magnetics, Vol. 32, No. 3, pp. 1767 1772, 1996.

(10) T.Yamaguchi, S.Shishida, S.Tohyama, Y.Soyama, H.Hosokawa, H.Ohsawa, H.Numasato, T.Arai, K.Tsuneta, and H. Hirai: Mode switching controller with initial value compensation for disk drive servo control, Control Engineering Practice, Vol. 5, no.11, pp. 1525-1532, 1997.

(11) J. Ishikawa, Y. Yanagita, T. Hattori, and M. Hashimoto: Head positioning control for low sampling rate systems based on two degree-of-freedom control, IEEE Trans. on Magnetics, Vol. 32, No. 3, pp. 1787-1792, 1996.

(12) R. Oboe and M. Federico: Initial value compensation applied to disturbance observerbased servo control in HDD, AMC2002, pp. 34-39, 2002.

(13) A. Okuyama, T. Hamaguchi, K. Watanabe, T. Horiguchi, K. Shishida, and Y. Nishimura: A design method - based on a shock response spectrum analysis - for reducing acoustic noise of a seeking control system in a hard disk drive, Springer-Verlag Microsystem Technologies, Vol. 9, No. 8, pp. 573-580, 2003.

(14) Y. Li, Y. Sun, C. Smith, L. Guo, and W. Guo: Optimization of initial value compensation for settle control in hard disk drives, Asia-Pacific Magnetic Recording Conference SV01-01/02, 2004.

(15) J. Paxman and G. Vinnicombe: optimal transfer schemes for switching controllers, Proceedings of the 39th IEEE Conf. on Decision and Control, pp. 1093-1098, 2000.

(16) M. Johansson: Optimal initial value compensation for fast settling times in modeswitching control systems, Proceedings of the 39th IEEE Conf. on Decision and Control, pp. 5137-5142, 2000. 